\title{
Monte Carlo estimation of chance performance for the radial arm maze
}

\author{
DAVID A. ECKERMAN \\ University of North Carolina, Chapel Hill, North Carolina 27514 and \\ Health Effects Research Laboratory, U.S. Environmental Protection Agency \\ Research Triangle Park, North Carolina 27711
}

\begin{abstract}
The radial arm maze is now widely used to assess spatial control of behavior. Determination of chance performance on the maze may be made difficult, however, because of response biases. Simulation of arm selection under various biases was used to provide several estimates of chance accuracy of arm selection. These estimates emphasize the type and degree of the problem in characterizing chance accuracy and may be useful to others in arriving at a relevant estimate.
\end{abstract}

The radial arm maze, only recently introduced into the psychological literature (Olton \& Samuelson, 1976) has already been extensively used to assess spatial control of behavior and spatial memory. Rats (see Olton, 1979, for current review), mice (Rodier, Reynolds, \& Roberts, 1979; MacPhail, Gordon, \& Moore, Note 1), and even snakes (Kunka, Bernstein, \& Kubie, Note 2) demonstrate spatial control of behavior with the device. Further, disruptions of this control by central nervous system lesions (Jarrard, 1978; Olton, Walker, \& Gage, 1978; Olton \& Werz, 1978; Rodier et al., 1979) and by scopolamine (Eckerman, Gordon, Edwards, MacPhail, $\&$ Gage, in press) show that performance on the radial arm maze is a sensitive baseline for assessing mechanisms of spatially controlled behavior.

In evaluating performance on the radial arm maze, accuracy of selection of arm is typically compared to an assumed chance level of accuracy (e.g., Olton, 1978). The commonly used estimate is unfortunately inappropriate in many cases, however. This report offers a means of improving the estimate of chance performance in such cases, by computer simulation of accuracy under several models of chance arm selection.

The radial arm maze is typically used in the following manner. The subject is placed on the center platform of the (usually elevated) maze, from which a number of arms (e.g., 4,8 , or 17) radiate, as do spokes from a hub. A bit of food is placed at the far end of each of these arms before the trial. The subject is then left on the maze until all bits of food are collected or until a fixed time has elapsed.

With relatively little training (5 to 10 trials, usually given one per day), rats develop highly accurate and efficient performance, avoiding reentry to arms from which food has already been collected. Such perfor-

This work was supported by the U.S. Environmental Protection Agency and by Training Grant MH-14269 from the National Institute of Mental Health. Reprints may be obtained by writing the author at the Department of Psychology, University of North Carolina, Chapel Hill, North Carolina 27514. mance seems to be controlled by extramaze visual cues that distinguish the various locations rather than by intramaze cues (Olton \& Collison, 1979; Zoldeck \& Roberts, 1978). The order of selections varies from trial to trial and subject to subject, implying that accurate performance is not simply a result of learning a particular route through the maze (Olton \& Samuelson, 1976; Eckerman et al., in press). The best characterization of the performance in many studies appears to be that rats select arms more or less unsystematically but choose arms without replacement (Olton, 1978).

In some studies, however, the selections have been systematic (e.g., Olton, Collison, \& Werz, 1977; Eckerman et al., in press, Experiment 1), and, in many studies, the selection pattern has not been described. When noted, the pattern has taken the form: select the neighboring arm clockwise with probability $\mathrm{x}$, select the arm two away clockwise with probability $y$, etc. This type of pattern is shown by an unequal frequency of selections one away, two away, etc., from the arm just exited. When this distribution is peaked and unimodal, the performance may be described as conforming to a simple response algorithm (Olton \& Samuelson, 1976). Such an algorithm does not select arms on the basis of whether food has or has not already been removed and cannot, therefore, be taken as evidence of spatial memory. To demonstrate spatial memory, accuracy must exceed that which would be produced by selecting arms strictly according to their location relative to the arm just exited. I propose below one means of arriving at an appropriate chance accuracy level.

The measure of chance accuracy commonly used is derived from treating each selection in a trial as random and calculating how many new arms would be chosen in M selections. As Olton (1978) pointed out, this then becomes an example of the "classical occupancy problem." The probability of entering $\mathrm{N}$ new arms in the first eight selections (on an eight-arm maze), given this procedure, are those shown as column 1 of Table 1 . 
Table 1

Probability of Choosing Exactly N Different Arms in Eight Selections

\begin{tabular}{lccccc}
\hline & & & & \multicolumn{2}{c}{ Bias from } \\
$\mathrm{N}$ & Random & $\begin{array}{c}\text { Random } \\
\text { Simula- } \\
\text { tion }\end{array}$ & $\begin{array}{c}\text { No } \\
\text { Immediate } \\
\text { Repeats }\end{array}$ & $\begin{array}{c}\text { Eckerman et al. } \\
\text { Experi- } \\
\text { ment 1 }\end{array}$ & $\begin{array}{c}\text { Experi- } \\
\text { ment 2 }\end{array}$ \\
\hline 8 & .0024 & .0040 & .0080 & .0075 & .0080 \\
7 & .0673 & .0695 & .1180 & .1900 & .1350 \\
6 & .3194 & .3335 & .4320 & .4425 & .4250 \\
5 & .4200 & .3990 & .3635 & .2935 & .3550 \\
4 & .1680 & .1795 & .0755 & .0510 & .0770 \\
3 & .0168 & .0135 & .0033 & .0025 & .0000 \\
2 & .0004 & .0010 & .0000 & .0000 & .0000 \\
1 & .0000 & .0000 & .0000 & .0000 & .0000 \\
Mean N & 5.26 & 5.28 & 5.61 & 5.78 & 5.65 \\
SD & .89 & .90 & .83 & .84 & .83 \\
Median N & 5.74 & 5.76 & 6.13 & 6.35 & 6.16 \\
\hline
\end{tabular}

Mean chance accuracy is usually taken to be 5.3 new arms in 8 selections.

Determination of chance accuracy where selections are biased becomes difficult to do except by Monte Carlo simulation. I proceeded as follows. A computer program allowed rapid "draws" of successive selections in the simulation. The arms chosen for selection $\mathrm{M}$ of a "trial" were determined probabilistically by discarding draws $\mathrm{i}$ away with probability $\mathrm{x}_{\mathrm{i}}$ and redrawing another arm (which also might be discarded, etc.) until a choice was set. By selecting an array of probabilities of rejection for arms over the range -3 to +4 away from the arm just exited, choices could be generated to characterize selections resulting from "blindly" following that particular response bias pattern. Counting the number of new arms $(\mathrm{N})$ chosen in the $\mathrm{M}$ selections produced the accuracy measure for that trial. Probabilities of $\mathrm{N}$ new selections were estimated from accuracy obtained on at least 1,000 "trials."

To validate the simulation procedure, a simulation was carried out with zero probability of rejecting any selection (i.e., random or unbiased choice). Column 2 of Table 1 shows that probabilities generated through simulation closely approximated those expected (column 1). The simulation procedure thus appears valid.

The simulations of column 3 were obtained with a response bias that is very common on the radial arm maze, namely, the subject did not choose the arm just exited (see Olton \& Samuelson, 1976). If these immediate repeat choices are eliminated, but arm choices are otherwise random, the mean chance number of new arms entered rises by .33 arms to 5.61 .

Eckerman et al. (in press, Experiment 1) found that selections were biased toward selection of a neighboring arm as well as against selection of the immediately prior one. Entering the response bias distribution they found (see Table 2), the data fell as in column 4 of the tablewith the mean number of new arms selected falling quite close to 6. In their Experiment 2, Eckerman et al. (in press) found a similar but lesser bias. When the reduced bias was entered into the simulation, chance accuracy decreased as shown in column 5 of Table 1 .

The level best taken to represent chance accuracy clearly depends upon what bias is present in selection of arms. Inasmuch as the observed bias leads the next selection away from areas of the maze already selected, the level of chance accuracy will rise above "column 1" levels even when there is no direct control over behavior by which arms nave or have not been entered previously (i.e., spatial memory).

It is noteworthy that published data from the radial arm maze to date all show accuracies higher than those in Table 1. There is, therefore, no concern over the validity of these reports. Olton and Samuelson (1976), however, refer in passing to an unpublished study showing that rats selected six new arms of the eight selecttions, indicating "a strong disposition not to repeat choices to arms." Whether the rats' selections did or did not indicate such a disposition depends on the type and degree of bias in the selections. It is hoped that this report will aid researchers in more carefully investigating such patterning in order to avoid future errors of interpretation.

I have been concerned here with estimating the number of new arms selected in the first $M$ choices. Roberts (1979) recently utilized another measure of accuracy-the number of selections required to obtain all bits of food. This measure has somewhat different properties. With completely random selection, computer simulation of the median number of selections required to enter all eight arms is 20.63 (the median is used since the distribution is skewed). If immediate reentries are eliminated, the median drops to 18.18. Bias obtained by Eckerman et al. (in press, Experiment 1) produced a further drop to 15.52. All these values, however, are nearly double the number of selections trained subjects typically make. This approach demonstrates spatial memory without the ceiling effect encountered by the more common measure, the number of new choices in the first $M$ selections. This method does, however, require that the trial continue until all bits of food are collected. It is therefore more time-consuming and is inappropriate when procedures are used that reduce the number of selections made (e.g., drug administration).

Another measure often utilized in characterizing accuracy on the radial arm maze is the percentage (averaged across subjects and/or trials) of first selections,

Table 2

Probability of Choosing Arms N Away from Prior Selection

\begin{tabular}{lccccccccc}
\hline & \multicolumn{3}{c}{ Nonpreferred Side } & & \multicolumn{4}{c}{ Preferred Side } \\
\cline { 2 - 7 } \cline { 7 - 10 } & -3 & -2 & -1 & 0 & & 1 & 2 & 3 & 4 \\
\hline Experiment 1 & .04 & .04 & .06 & .02 & .45 & .23 & .10 & .06 \\
Experiment 2 & .06 & .09 & .08 & .02 & .22 & .26 & .17 & .10 \\
\hline
\end{tabular}

Note-From Eckerman et al., in press 
second selections, etc., that are correct (see Olton \& Samuelson, 1976). This function starts at $100 \%$ for the first selection of a trial, since that selection is by necessity to a new arm. The percentage then typically falls across successive selections within the trial (e.g., Eckerman et al., in press, Figure 1). The values on the function can be corrected for chance by finding how many new arms were available to the subject at each selection. A "chance" level of accuracy is usually then taken to be the number of new arms available divided by the total number of arms. Obtained percent accuracy is expressed relative to the chance level (see Olton \& Samuelson, 1976). The logic of the present argument suggests that each still-new arm not enter an equal amount to the estimation of chance for a rat, but that each still-new arm be weighted by a preference factor determined for that rat from past selection patterns. A working formula and normative data for this correction, however, is beyond the scope of the present report.

The specific recommendation of this report is as follows. When considering the appropriate "chance accuracy" against which to compare an obtained accuracy for performance on the radial arm maze, take the response bias into account. One solution is to enter the response bias into otherwise random selections of arms in a computer simulation. By analyzing the number of new arms selected in such simulations, a chance accuracy may be determined which takes the obtained response bias into account.

\section{REFERENCE NOTES}

1. MacPhail, R. C., Gordon, W. A., \& Moore, J. W. Personal communication, 1979.

2. Kunka, M. G., Bernstein, I. G., \& Kubie, J. L. Short-term spatial memory in garter snakes. Paper presented at the meeting of the Eastern Psychological Association, Philadelphia, April 1979.

\section{REFERENCES}

Eckerman, D. A., Gordon, W. A., Edwards, J. D., MacPhail, R. C., \& GAGE, M. I. Effects of scopolamine, pentobarbital, and amphetamine on radial arm maze performance in the rat. Pharmacology, Biochemistry, and Behavior, 1979, in press.

JARRARD, L. E. Selective hippocampal lesions: Differential effects on performance by rats of a spatial task with preoperative versus postoperative training. Journal of Comparative and Physiological Psychology, 1978, 92, 1119-1127.

Olton, D. S. Characteristics of spatial memory. In S. H. Hulse, W. K. Honig, \& H. Fowler (Eds.), Cognitive aspects of animal behavior. Hillsdale, N.J: Erlbaum, 1978.

Olton, D. S. Mazes, maps and memory. American Psychologist, 1979, 34, 583-596.

Olton, D. S., \& Collison, C. Intramaze cues and "odor trails" fail to direct choice behavior on an elevated haze. Animal Learning \& Behavior, 1979, 7, 221:223.

Olton, D. S., Collison, C., \& Werz, W. A. Spatial memory and radial arm maze performance by rats. Learning and Motivation, 1977, 8, 289-314.

Olton, D. S., \& Samuelson, R. J. Remembrance of places passed: Spatial memory in rats. Journal of Experimental Psychology: Animal Behavior Processes, 1976, 2, 97-116.

Olton, D. S., Walker, J. A., \& GAge, F. H. Hippocampal connections and spatial discrimination. Brain Research, 1978, 139, 295-308.

Olton, D. S., \& Werz, M. A. Hippocampal function and behavior: Spatial discrimination and response inhibition. Physiology and Behavior, 1978, 20, 597-605.

ROBE RTS, W. A. Spatial memory in the rat on a hierarchical maze. Learning and Motivation, 1979, 10, 117-140.

Rodier, P. M., Reynolds, S. S., \& Roberts, W. N. Behavioral consequences of interference with CNS development in the early fetal period. Teratology, 1979, 19, 327-336.

Zoldeck, L., \& Roberts, W. A. The sensory basis of spatial memory in the rat. Animal Learning \& Behavior, 1978, 6, 77-81.

(Received for publication November 19, 1979.) 